\title{
Brunner's gland adenoma - a rare cause of iron deficiency anaemia
}

\author{
M. Priyanthi Kumarasinghe ${ }^{1}$, K. L. Fernando ${ }^{2}$ \\ The Ceylon Journal of Medical Science 1997; 40: 25-27
}

\section{Summary}

A 35 year old female who had epigastric pain and iron deficiency anaemia for several months was diagnosed to have a Brunner's gland adenoma. Upper gastro-intestinal endoscopy (UGIE) performed twice and barium meal follow through failed to reveal any abnormality initially. The third UGIE showed a polyp in the junction between the 1st and 2nd parts of the duodenum. Histology of the polyp showed features of a Brunner's gland adenoma.

Brunner's gland adenoma is a rare tumour of the duodenum. Endoscopic biopsy diagnosis is often difficult. Haemorrhage and obstruction, both recognised complications of a Brunneroma were evident in this patient. Recurrent epigastric pain was most probably due to obstruction. Haemorrhage was severe enough to cause iron deficiency anaemia.

Patient is free of symptoms and signs six months after successful resection of the polyp.

\section{Introduction}

Duodenal tumours are rare; brunneromas represent about $10 \%$ of them. Brunner's gland adenoma also known as brunneroma or polypoid hamartoma can clinically mimic a malignancy. Some believe that these are true neoplasms while others believe that they are hamartomas $(1,2)$. The most frequent site is the junction between the first and the second parts of the duodenum. These lesions can be the cause of duodenal obstruction or haemorrhage. An unequivocal diagnosis by endoscopic biopsy is not possible (3). Complete resection either endoscopically or by surgery is the treatment.
Rare cases of carcinomas associated with the Brunner's gland have been reported (4).

\section{Case history}

A 35 year old female presented with epigastric pain and anaemia. Her haemoglobin level at presentation was $7.6 \mathrm{~g} / \mathrm{dl}$ with the blood picture showing features of iron deficiency. Total iron was below normal, total iron binding capacity was abnormal and serum ferritin was below normal. Upper gastro-intestinal endoscopy (UGIE) done on 2 occasions and barium meal follow through did not show any abnormality. Stools examination for occult blood was negative on three occasions. A third UGIE was done as her signs and symptoms persisted and a polypoid lesion was detected in the 2nd part of the duodenum. The endoscopic appearance was suspicious of a malignancy. The endoscopic biopsy showed abnormal duodenal mucosal glands with irregularity and dilatation without malignant features. A benign adenoma or abnormal duodenal mucosa overlying a sub mucosal lesion was suspected on histology.

A laparotomy with possible pancreatoduodenectomy was planned with preparation for a frozen section and per-operative imprint cytology. CT scan done preoperatively was normal. At laparotomy a smooth, lobulated polyp with a focally haemorrhagic surface was found at the junction between the first and second parts of the duodenum. As there was no macroscopic evidence of malignancy a polypectomy with a sleeve resection of the duodenal wall at the base was done.

Pathologic examination revealed a smooth lobulated polyp measuring $4 \times 3 \times 2 \mathrm{cms}$ with a

1 Associate Professor of Pathology, Department of Pathology, Faculty of Medicine, University of Colombo.

2 Consultant Surgeon and Surgical Gastroenterologist, 30, Sapumal Place, Circular Road, Rajagiriya. 
$2 \mathrm{~cm}$ long stalk and an area of surface haemorrhage (Figure 1). The haemorrhagic area was thought to be the result of endoscopic biopsy. The stalk of the polyp was well developed with no evidence of induration at the base.

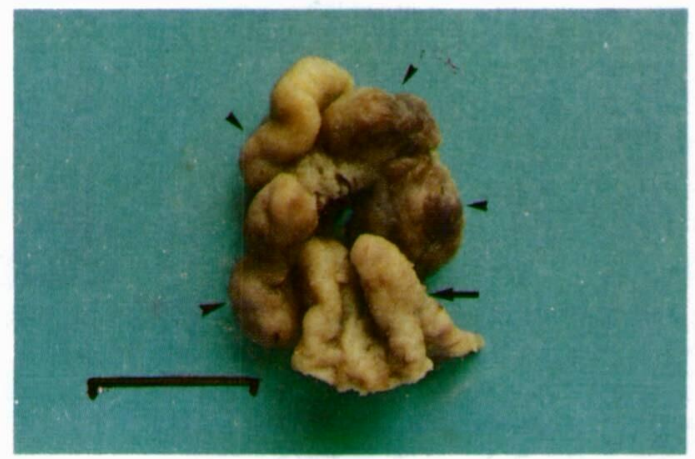

Figure 1

The polypoid tumour covered with the duodenal mucosa (arrowheads), the stalk (arrow) and the duodenal wall at the base.

$(\mathrm{Bar}=3 \mathrm{~cm})$

Haematoxylin and eosin stained sections showed the classical picture of a Brunner's gland adenoma composed of nodular proliferation of morphologically normal Brunner's glands and ducts (Figure 2).

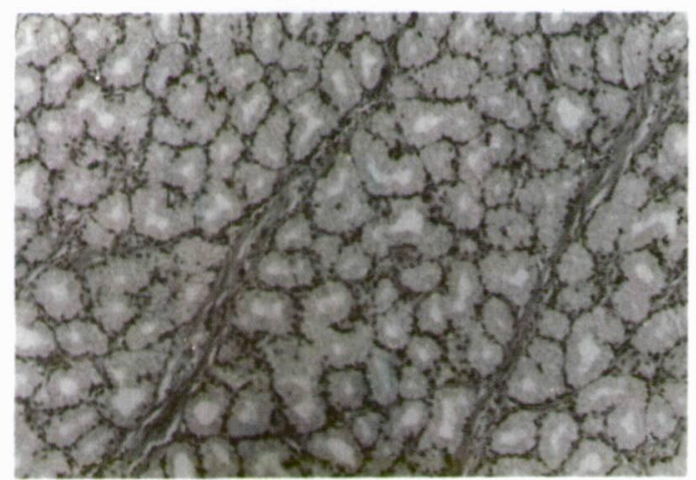

Figure 2

The proliferation of Brunner's glands (H \& E x 100)

Post operative period was uneventful. The patient is free of symptoms and signs six months after resection of the polyp.

\section{Discussion}

The benign epithelial tumour characterised by proliferation of morphologically normal Brunner's glands is given a variety of names; polypoid hamartoma, Brunner's gland adenoma and Brunneroma. The exact pathological nature is uncertain as some believe that this is not a true neoplasm but a hyperplasia of Brunner's glands. Focal, multifocal and diffuse forms occur. Melaena and obstruction can be caused by the tumour. Carcinomatous transformation has been described.

UGIE performed on two occasions in this patient did not reveal any abnormality. The duodenal lesion was probably overlooked partly because duodenal neoplasms are rare. The other possible reason is that the polyp with the long stalk may have moved to the third part of the duodenum preventing visualization through the endoscope. A definite histologic diagnosis on the endoscopic biopsy is difficult as the polyp consists of morphologically normal Brunner's glands.

The chronic epigastric pain in this patient may have been due to partial obstruction while iron deficiency anaemia was clearly due to haemorrhage. A duodenal neoplasm should be considered as a rare but possible cause of significant gastro-intestinal haemorrhage. In this patient the tumour was confirmed to be a rare Brunner's gland adenoma. The associated haemorrhage was severe enough to cause iron deficiency anaemia in this patient.

This work was carried out at the Department of Pathology, Faculty of Medicine, Colombo and Nawaloka Hospital, Colombo.

\section{References}

1. Juwan Rosai. Ackerman's Surgical Pathology, 8th edition, St. Louis, CV Mosby and Company, 1996; page 683.

2. Chandrasoma P, de Silva S. Giant duodenal hamartoma consisting mainly of Brunners glands. American Journal of Surgery 1977; 133:240-243. 
3. Hardt M, Kruis W, Edit S. A rare cause of upper gastrointestinal haemorrhage: large adenoma of Brunner's glands.

Z-Gastroenterology 1994; 32:589-591.

4. Itsuno M, Makiyama K, Omagari K, Tanaka T, Hara K et al. Carcinoma of duodenal bulb arising from the Brunner's gland. Gastroenterology Japan 1993; 28:118-125.

5. Matsui K, Kitagawa M. Biopsy study of polyps in the bulb. American Journal of Gastroenterology. 1993; 88:253-257. 